CRÍTICA, Revista Hispanoamericana de Filosofía

Vol. XXXII, No. 96 (diciembre 2000): 43-57

\title{
KOLMOGOROV, HEYTING AND GENTZEN ON THE INTUITIONISTIC LOGICAL CONSTANTS*
}

\author{
Gustavo Fernández Díez \\ Facultad de Filosofía \\ Universidad de Murcia
}

\section{Kolmogorov's Interpretation: an Interpretation in Terms of Mathematical Problems}

Kolmogorov's 1932 paper is the first published attempt to give an explicit and systematic account of all the intuitionistic logical operators.

In this paper Kolmogorov outlines an interpretation that is patently verificationist. He argues that it would be a mistake to try to give an interpretation of intuitionistic logic based on the notion of 'truth'; instead, he proposes the notions of 'problem' and 'solution to a problem':

In addition to theoretical logic, which systematizes a proof schemata for theoretical truths, one can systematize a proof schemata for solutions to problems [...].

In the second section [of the paper], assuming the basic intuitionistic principles, intuitionistic logic is subjected to

* The contents of this paper, in a somewhat different form, are part of my doctoral dissertation at the London School of Economics and Political Science, written under the direction of Professors Colin Howson and Moshe Machover. The research was supported, at various stages, by the British Council, the British Academy, the Spanish Ministry of Education and Caja de Ahorros del Mediterráneo. I would like to thank as well an anonymous referee of Crítica for his extensive comments and criticisms on an earlier draft of this paper. 
a critical study; it is thus shown that it must be replaced by the calculus of problems, since its objects are in reality problems, rather than theoretical propositions. (Kolmogorov (1932), p. 58) ${ }^{1}$

As we shall see immediately, Kolmogorov defines what is to be the solution of a complex problem in terms of solutions of its logical components, depending on what the main logical operator is. By doing so, he establishes the general form of the so-called 'verificationist interpretation': an inductive compositional definition of the notion of 'solution to a problem'. Later versions of the verificationist interpretation shall use the concept of 'proof' rather than that of 'solution to a problem', but the spirit of the definition will remain the same. Also, in so doing, Kolmogorov is giving the first general definition of the concept of "constructive proof', although he does not present it under this label.

Kolmogorov was anticipated in several respects by Heyting's papers of 1930 and 1931. In particular, in these papers Heyting also outlines the essence of the verificationist interpretation and uses it to explain the intuitionistic use of negation and disjunction. However, Kolmogorov's work was independent of that of Heyting. Indeed, at the end of his 1932 paper he included a footnote - added at proofreading stage - in which he credits the similarity between his interpretation and that of Heyting (1931), which had appeared recently; this footnote reads: "This interpretation of intuitionistic logic is intimately related to the ideas that $\mathrm{Mr}$ Heyting has advanced in the latest volume of Erkenntnis [...]" (Kolmogorov (1932), p. 65, fn. 17).

Heyting, on the other hand, in his 1934 book, acknowledges the independence - as well as the similarity - of Kolmogorov's interpretation in terms of problems, and

1 As there is no English translation of this paper, I have used my own in all quotations here cited. 
adopts it himself to explain the meaning of the intuitionistic logical constants and to give semantic motivations to several logical theorems (Heyting (1934), pp. 17-23). ${ }^{2}$

As for Brouwer, the founder of intuitionism and the person who 'invented' these logical operators, he did not have a great interest in mathematical logic, which he considered subordinate to mathematics (in fact, a mere description of regularities in mathematical practice), ${ }^{3}$ and perhaps for this reason he never committed himself to giving a precise explication of these operators. His 'revolutionary' conception of the logical constants has to be traced in the use he made of them in his proofs of intuitionistic theorems, and in the comments accompanying those proofs, something which I have done at some length in (2000a).

\section{Kolmogorov's Interpretation of the Connectives}

Kolmogorov's interpretation of the intuitionistic connectives is as follows:

$A \wedge B$ is "the problem of "solving both $A$ and $B$ " ";

$A \vee B$ is "the problem of "solving at least one of $A$ and $B$ " "; $A \rightarrow B$ is "the problem of "solving $B$ supposing that the solution to $A$ is given" ";

and finally,

2 Since there is no English version of this book, I have used my own translation in all quotations taken from it. For this purpose I have used the French expanded edition, Les fondements des Mathématiques. Intuitionisme. Théorie de la Démonstration, Gauthier-Villars, Paris, 1955. Notice, however, that all the passages here quoted belong to the German 1934 edition (the additions to the original text being clearly marked as such in the French version).

3 Cf., e.g., van Stigt (1990), pp. 224-238. 
$\neg A$ is "the problem of "obtaining a contradiction supposing that the solution to $A$ is given" ". (Kolmogorov (1932), pp. $59-60)^{4}$

With respect to the conditional, Kolmogorov explains: "or, what amounts to the same, "to carry the solution of $B$ back to the solution of $A$ " " (Kolmogorov (1932), p. 59). According to this, what he has in mind is a partial solution or solution-schema of $B$, which would become a full solution if complemented with a solution of $A$. In other words: a solution of $B$ with premise $A$. And this implies a tacit appeal to a hypothetical solution of $A$, to which the proof of $B$ would be carried back to.

Negation stands exactly in the same situation. In fact, a negation statement $\neg A$ appears as a special kind of conditional statement, $A \rightarrow B$, in a case where $B$ is a contradiction. Kolmogorov does not explain, however, what he understands by 'contradiction' (Widerspruch), which in turn would have to be defined without using negation, on pain of circularity.

\section{Kolmogorov's Interpretation of the Quantifiers}

Kolmogorov seems more concerned with the interpretation of intuitionistic propositional logic than with predicate logic; in fact, he gives only the propositional part of the formal axiom system ('calculus of problems'). ${ }^{5}$ However, after explaining the meaning of the connectives, he extends his interpretation in terms of problems to the universal quantifier:

Generally speaking, if $x$ is a variable (of the type desired) and $P(x)$ is a problem whose meaning depends on the variable $x$,

${ }^{4}$ I use my own choice of notation in this and the subsequent quotations.

${ }^{5}$ Cf. Kolmogorov (1932), pp. 61-62. 
then $\forall x P(x)$ is the problem of "indicating a general method for the solution of $P(x)$ for each particular value of $x$ ". This should be understood like this: to solve the problem $\forall x P(x)$ means to be in a position to solve the problem $P(a)$ for each given value $a$ of $x$, after a series of steps given in advance (before the choice of $a$ ). (Kolmogorov (1932), p. 60)

Following this, Kolmogorov does not give the interpretation of the existential quantifier, as we would expect, since intuitionistically the existential quantifier cannot be defined using the universal and negation; but elsewhere in the paper he gives ample explanations of the meaning of existential claims in intuitionistic mathematics, and in particular, of the central point concerning them: that the person who makes the claim must be able to indicate a particular instance of it.

In any case, it is very easy to apply the preceding definition to the intuitionistic $\exists$, thus (with $P(x)$ as before):

the solution to $\exists x P(x)$ is the indication of a particular object $a$ plus a solution to $P(a)$.

Heyting himself, in his exposition of Kolmogorov's interpretation, includes basically this definition of $\exists$ as a straightforward extension of Kolmogorov's way of explaining the meaning of the other logical constants (Heyting (1934), p. 21).

\section{The Basics of Heyting's Interpretation}

Heyting's interpretation in its standard form does not appear until his best-known textbook Intuitionism: An Introduction (1956). It is in this book that we first find his own systematic explanation of all the intuitionistic logical operators, entirely based on the notions of 'proof' and 'assertability conditions'. 6

${ }^{6}$ Cf. sections 6 and 7 below. 
The basics of this definition, however, are already clear in his 1930 and 1931 papers, and in his 1934 book. In those works, Heyting openly defends the verificationist point of view, uses it to define several connectives, and comments positively on the variant of Kolmogorov.

This is Heyting's defence of verificationism, arising from the constructive standpoint:

Here is thus an important result of the intuitionistic critique: the idea of an existence of the mathematical entities outside our mind should not enter into the demonstrations. I think that even the realists, while continuing to believe in the transcendent existence of mathematical entities, should recognize the importance of knowing in what way mathematics can be built without using this idea.

For the intuitionists, mathematics constitutes a magnificent edifice built by human reason. Perhaps they would do better to avoid entirely the word "to exist"; if they continue to use it nevertheless, it could not have, for them, any other sense than this of "having been built by reason". (Heyting (1930), p. 958; italics in the original. $)^{7}$

Consequently, mathematical statements have to be interpreted in a non-realist way:

A mathematical proposition expresses a certain expectation. For example, the proposition, "Euler's constant $e$ is rational", expresses the expectation that we could find two integers $n$ and $m$ such that $e=n / m$. Perhaps the word "intention", coined by the phenomenologists, expresses even better what is meant here. (Heyting (1931), p. 58)

There is a criterion by which we are able to recognize mathematical assertions as such. Every mathematical assertion can be expressed in the form: "I have effected the construction $c$ in my mind." (Heyting (1956), pp. 18-19)

7 As there is no English translation of this paper, I have used my own in all quotations here cited. 
In addition to this, Heyting makes it quite clear that, for him, to effect the construction required by a mathematical statement, and to give a proof of it, are one and the same thing:

The demonstration of a proposition consists in the realization of the construction that it requires. (Heyting (1934), p. 17)

[A] mathematical proposition $A$ always demands a mathematical construction with certain properties; it can be asserted as soon as such a construction has been carried out. We say in this case that the construction proves the proposition $A$ and call it a proof of $A$. (Heyting (1956), p. 98)

[E]very mathematical theorem is the expression of a result of a successful construction. The proof of the theorem consists in this construction itself, and the steps of the proof are the same as the steps of the mathematical construction. (Heyting (1958), p. 107)

And the way in which he defines the connectives at different places confirms this identification too; for example, in the case of disjunction:

$A \vee B$ signifies that intention which is fulfilled if and only if at least one of the intentions $A$ and $B$ is fulfilled. (Heyting (1931), p. 59)

And

$A \vee B$ can be asserted if and only if at least one of the propositions $A$ and $B$ can be asserted. (Heyting (1956), p. 97)

This identification between the construction required by a mathematical statement and the (constructive) proof of the same statement is wrong, as I show in my paper (2000). For example, in a theorem of the form

$$
\forall x(P(x) \rightarrow \exists y R(x, y))
$$


we might have invented a construction which appears to transform every object with property $P$ into a second one, related to the first by the relation $R$ (i.e., so that it appears that for each $a$ such that $P(a)$, our construction produces an object $b$ such that $R(a, b)$ ); and still, until we manage to prove that such a construction does work in general (for every object in the domain), we will not have a proof of the theorem. Even if that proof is eventually found, and therefore we get a confirmation that our tentative construction behaved as required, there would nonetheless be a difference between that construction alone, and the subsidiary proof that the construction works as required. In other words: there would be a difference between the construction which transforms every object $a$ such that $P(a)$ into an object $b$ such that $R(a, b)$, and that additional proof which shows that the construction in question does indeed such a transformation for any object $a$ in the domain. ${ }^{8}$

\section{Heyting and Kolmogorov}

On the other hand, as we know, Heyting also acknowledges the similarity between his proof interpretation and that of Kolmogorov's. As I said before, in his 1934 paper Heyting uses Kolmogorov's interpretation to motivate the intuitionistic rejection of various classical logical principles and the acceptance of others. Before doing this, he writes:

Kolmogorov [...] has proposed a similar conception [...]. He interprets this calculus as a calculus of problems. [...] he does not explicate this concept, which we could interpret as the request to effect a mathematical construction which satisfies certain conditions. (Heyting (1934), p. 17)

${ }^{8}$ For a more specialized example and further discussion, cf. my paper (2000) just mentioned, in particular section 3, pp. 411-412. 
Indeed, earlier in his 1930 paper, he had written: “A proposition [...] expresses a problem, or even better a certain expectation [...]" (Heyting (1930), p. 958). And later he will insist: "The older interpretations by Kolmogorov (as a calculus of problems) and Heyting (as a calculus of intended constructions) were substantially equivalent" (Heyting (1958), p. 107).

\section{Heyting's Interpretation of the Connectives}

In any case, in his 1956 book, Heyting takes the concept of 'provability' (or 'assertability conditions') as the basic notion of the whole definition, and in the same way do most authors afterwards: "It will be necessary to fix, as firmly as possible, the meaning of the logical connectives; I do this by giving necessary and sufficient conditions under which a complex expression can be asserted" (Heyting (1956), p. 97). And it is in these terms that he gives the interpretation of the connectives, as follows:

$A \wedge B$ can be asserted if and only if both $A$ and $B$ can be asserted.

$A \vee B$ can be asserted if and only if at least one of the propositions $A$ and $B$ can be asserted.

$A \rightarrow B$ can be asserted, if and only if we possess a construction $c$, which, joined to any construction proving $A$ (supposing that the latter be effected), would automatically effect a construction proving $B$.

And

$\neg A$ can be asserted if and only if we possess a construction $c$, which, from the supposition that a construction $A$ were carried out, leads to a contradiction. (Heyting (1956), pp. 97-98) 
The last but one clause refers to a construction which, as a matter of fact, if joined to a construction proving the antecedent $(A)$ would effect the consequent $(B)$. We could paraphrase it as 'a construction $c$ such that, for any construction $d$, if $d$ proves $A$ then $c(d)$ proves $B$ '. Hence the reference to hypothetical constructions is not essential. And the formulation of the 1934 paper just mentioned agrees entirely with it: " $A \rightarrow B$ then represents the intention of a construction which, from each demonstration of $A$, leads to a demonstration of $B$ " (Heyting (1934), p. 17). This shows that Kolmogorov's and Heyting's explanations of the intuitionistic conditional are not at all identical.

In contrast, the definition of negation appeals explicitly to a proof of a contradiction from premise $A$, that is: a hypothetical proof of a contradiction which would use as a premise the existence, hypothetical as well, of a construction proving $A$. Moreover, other definitions of negation that Heyting gives in different places are also of this form, e.g.: "The proposition ' $e$ is not rational' $[\ldots]$ signifies the expectation that one can derive a contradiction from the assumption that $e$ is rational" (Heyting (1931), p. 59).

This means that there is a subtle difference between Heyting's definition of the conditional and that of negation: the definition of negation uses the notion of 'proof from premises', and hence the notion of hypothetical proof, and is in this sense similar to Kolmogorov's. The definition of the conditional, on the other hand, merely requires a construction by means of which it is possible to produce an actual proof of the consequent, provided that we possess a proof of the antecedent.

It might seem that this difference is completely innocuous or devoid of interest. But this is not at all the case, as I show in my paper (2000), cited above: ${ }^{9}$ once we agree

${ }^{9}$ Cf. specially section 8, pp. 416-418. 
to define the proof of any conditional statement $A \rightarrow B$ as a proof of $B$ with premise $A$, we must immediately redefine all the other logical constants in terms of proofs from premises (e.g. in case $B$ is complex), and this brings about a number of problems which are not at all present if we do not use this notion, and which are rather difficult to solve. (For example, a proof of $A \rightarrow(C \vee D)$ would be described, in terms of proofs from premises, as a proof of $C \vee D$ from premise $A$. But we cannot in turn define a proof of $C \vee D$ from premise $A$ as 'a proof of $C$ from premise $A$ or a proof of $D$ from premise $A$ ', because that would legitimate the law of distribution of $\rightarrow$ into $\vee$, which is strongly non-constructive, and certainly not deducible in the intuitionistic propositional calculus.)

\section{Heyting's Interpretation of the Quantifiers}

Heyting's interpretation of the quantifiers is as follows:

$\vdash \forall x P(x)$ means that $P(x)$ is true for every $x$ in $D$ [the domain]; in other words, we possess a general method of construction which, if any element $a$ of $D$ is chosen, yields by specialization the construction $P(a)$.

And

$\exists x P(x)$ will be true if and only if an element $a$ of $D$ for which $P(a)$ is true has actually been constructed. (Heyting (1956), p. 102)

It is remarkable that in the latter clause Heyting does only require that an instance of $P(x)$ is produced, but not that it is shown to be such an instance. In general (with $P(x)$ complex) this will not be evident, and will ask as well for a separate proof. The requirement of that additional proof is embodied in Gentzen's interpretation, as we shall see immediately. 
8. Gentzen's Natural Deduction Rules: Intuitionistic Version

When Gentzen presented his natural deduction calculus, and in particular the intuitionistic version, he wrote the following: "The introductions [the introduction rules] represent, as it were, the 'definitions' of the symbols concerned, and the eliminations are no more, in the final analysis, than the consequences of these definitions" (Gentzen (1935), p. 80). Indeed, he had intended to create a formal system which came as close as possible to actual mathematical reasoning (Gentzen (1935), p. 74); hence the way in which the rules governing each logical constant were given, and in particular, the introduction rules, would have to be immediately connected with its intuitive meanings.

Gentzen's introduction rules are well-known. According to them,

a proof of $A \wedge B$ is given by a proof of $A$ plus a proof of $B$;

a proof of $A \vee B$ is given either by a proof of $A$ or by a proof of $B$;

a proof of $A \rightarrow B$ is a proof of $B$ from premise $A$;

a proof of $\neg A$ is a proof of $A \rightarrow \perp$, where $\perp$ is any false statement;

a proof of $\forall x P(x)$ is a proof of $P(y)$ for a critical variable $y$ which does not occur in $\forall x P(x)$ or in any non-discharged premise; and

a proof of $\exists x P(x)$ is a proof of $P(t)$ for some term $t .^{10}$

Gentzen's contribution is important even if his main concern was not that of giving a semantic explanation, because he makes a clear and explicit use of the notion of "proof

10 Cf. Gentzen (1935), pp. 77-79. 
from premises' to define both negation and the conditional, and introduces a definition of the universal quantifier somehow connected to it: the definition in terms of "proofs with free variables', which has later been adopted by other authors, such as Per Martin-Löf or Göram Sundholm. ${ }^{11}$

Notice, however, that Kolmogorov's interpretation explicitly appeals to a 'general method' for solving the instances of the universal statement. Hence his interpretation is clearly divergent from that of Gentzen's at this point, and similar to that of Heyting's.

As before, it might seem that the difference between a 'general method' to prove $P(x)$ for each $x$ and a 'freevariable proof' of $P(x)$ is unimportant, but this is not so, as $I$ argue in my paper (2000): $:^{12}$ once we define the proof of $\forall x P(x)$ as a free-variable proof of $P(x)$, we have to redefine as well all the other logical constants in terms of proofs with free variables (in case $P(x)$ is complex), and the way to do this is quite problematic.

\section{REFERENCES}

Fernández Díez, G. (2000), "Five Observations Concerning the Intended Meaning of the Intuitionistic Logical Constants", Journal of Philosophical Logic, no. 29, pp. 409-424.

— (2000a), "Brouwer's Understanding of the Logical Constants", Indian Philosophical Quarterly, no. 27, pp. 215-228.

Gentzen, G. (1935), "Untersuchungen über das logische Schliessen" (I and II), Mathematische Zeitschrift, no. 39, pp. 176210 and 405-431. Quoted here by the English translation in his Collected Papers, ed. by M.E. Szabo, North-Holland, Amsterdam, 1969, pp. 69-131.

11 Cf. Martin-Löf (1985), p. 54, (1987), pp. 410-412; Sundholm (1986), p. 491.

12 Cf. specially section 10 , pp. 419-420. 
Heyting, A. (1930), "Sur la logique intuitionniste", Académie Royale de Belgique: Bulletin de la Classe des Sciences (5), no. 16, pp. 957-963.

- (1931), "Die intuitionistische Grundlegung der Mathematik", in Erkenntnis, no. 2, pp. 106-115. Quoted here from the English translation in P. Benacerraf and H. Putnam (eds.), Philosophy of Mathematics: Selected Readings, 2nd. rev. ed., Cambridge University Press, New York, 1983, pp. 52-65.

- (1934), Mathematische Grundlagenforschung. Intuitionism. Beweistheorie, Springer, Berlin, 1934.

_- (1956), Intuitionism: An Introduction, North-Holland, Amsterdam (3rd. rev. ed. in 1971).

— (1958), "Intuitionism in Mathematics", in R. Klibansky (ed.), Philosophy in the Mid-Century: A Survey, vol. 1: Logic and Philosophy of Science, La Nuova Italia Editrice, Florence, pp. 101-115.

Kolmogorov, A.N. (1932), "Zur Deutung der intuitionistischen Logik", Mathematische Zeitschrif, no. 35, pp. 58-65.

Martin-Löf, P. (1985), "On the Meanings of the Logical Constants and the Justifications of the Logical Laws", Atti degli Incontri di Lògica Matemàtica, vol. 2, Scuola di Specializzazione in Lògica Matemàtica, Dipartimento de Matemàtica, Università di Siena, Siena, pp. 203-281. Quoted here by the reprint in the Nordic Journal of Philosophical Logic, no. 1 (1996), pp. 11-60.

(1987), "Truth of a Proposition, Evidence of a Judgement, Validity of a Proof", Synthese, no. 73, pp. 407-420.

Stigt, W.P. van (1990), Brouwer's Intuitionism, North-Holland, Amsterdam.

Sundholm, G. (1986), "Proof Theory and Meaning", in D. Gabbay and F. Guenthner (eds.), Handbook of Philosophical Logic, vol. 3: Alternatives to Classical Logic, Reidel, Dordrecht, pp. 471-506.

Recibido: 25 de octubre de 2000 


\section{RESUMEN}

Los primeros autores en elaborar definiciones semánticas de las constantes lógicas intuicionistas fueron Kolmogorov, Heyting y Gentzen, a comienzos de la década de 1930. Aunque sus explicaciones puedan a primera vista parecer equivalentes (y en concreto, por lo que respecta a Kolmogorov y Heyting, ellos mismos las percibieron como tales), el conciso estudio que presento en este artículo demuestra la existencia de diferencias sutiles pero nada triviales entre ellas, diferencias cuyas consecuencias filosóficas he explorado en detalle en otras publicaciones. 